\title{
Rapid impact of COVID-19 infection on semen quality: a case report
}

\author{
Parviz Gharagozloo $^{1} \wedge$, Stephanie Cartagena ${ }^{1} \wedge$, Aron Moazamian ${ }^{1} \wedge$, Joel R. Drevet $^{2} \wedge$, Stephen Somkuti ${ }^{3}$, \\ Robert John Aitken ${ }^{4} \wedge$ \\ ${ }^{1}$ CellOxess LLC, Ewing, NJ, USA; ${ }^{2}$ Université Clermont Auvergne, Clermont-Ferrand, France; ${ }^{3}$ Sincera Reproductive Medicine, Fort Washington, \\ PA, USA; ${ }^{4}$ Priority Research Centre for Reproductive Science for Reproductive Science, University of Newcastle, Callaghan, Australia \\ Correspondence to: Dr. Parviz Gharagozloo, PhD. CellOxess LLC, Ewing, NJ 08628, USA. Email: Info@celloxess.com.
}

\begin{abstract}
A unique opportunity to conduct a longitudinal analysis of semen quality in a male subject immediately before, during and after COVID-19 infection, has revealed new insights into the impact of this virus on male reproductive function. A moderate COVID infection that did not require hospitalization resulted in a state of azoospermia that persisted for 4 weeks. Given that the duration of spermatogenesis and epididymal sperm maturation in the human is 78 days, we calculate that a viral attack on the germ line was initiated at or before the patient was symptomatic and may have been signalled by a sudden reduction in sperm count and motility, several weeks earlier. Before the virus had been fully cleared, reinitiation of spermatogenesis occurred as evidenced by spermatozoa reappearing in the ejaculate exhibiting high levels of motility but significant levels of oxidative DNA damage as measured by a modified 8-OHdG assay protocol. These unique data indicate that even a moderate COVID-19 infection is capable of rapidly inducing a state of azoospermia that rapidly reverses as the infection wanes.
\end{abstract}

Keywords: Spermatozoa; COVID-19; semen quality; DNA damage; case report

Submitted Oct 21, 2021. Accepted for publication Dec 06, 2021.

doi: $10.21037 /$ tau-21-935

View this article at: https://dx.doi.org/10.21037/tau-21-935

\section{Introduction}

The COVID-19 pandemic has been responsible for the loss of over 4.5 million lives since it first broke out of China towards the end of 2019. Initially, it was the elderly or patients with pre-existing medical conditions such as asthma, diabetes, and heart disease who appeared to be particularly vulnerable to viral attack. However, subsequently, the highly infectious Delta variant has proven capable of infecting younger age groups and creating a range of morbidities including the disruption of male fertility $(1,2)$. The spike protein that gives the COVID-19 corona virus its name, is known to target ACE2 (angiotensin converting enzyme 2), which is highly expressed by several cell types in the testes including Leydig cells, Sertoli cells and the germ line (1). What little data is currently available, suggests that COVID-19 can be detected in the semen of a small proportion of patients and can certainly gain access to the testes. In this location, it induces both a reduction in testosterone levels and the disruption of semen quality, impacting all aspects of the latter including sperm motility, morphology, and count (3-6). However, there is a surprising degree of variability between studies, with some analyses reporting a very mild disruption of the semen profile (7) while others report azoospermia in a majority of patients (8). Some of this disparity may reflect shortcomings in published studies addressing the impact of COVID-19

^ ORCID: Parviz Gharagozloo, 0000-0003-3955-3297; Stephanie Cartagena, 0000-0001-8877-557X; Aron Moazamian, 0000-0002-1291-

4390; Joel R. Drevet, 0000-0003-3077-6558; Stephen Somkuti, 0000-0002-8388-6171; Robert John Aitken, 0000-0002-9152-156X. 
Table 1 Background data on donor semen quality in samples collected from 09/25/2018 to 12/05/2019

\begin{tabular}{|c|c|c|c|c|c|}
\hline Semen volume $(\mathrm{mL})$ & Sperm concentration $\left(10^{6} / \mathrm{mL}\right)$ & Total sperm count (millions) & Total motility (\%) & Rapid motility (\%) & 8-OHdG (\%) \\
\hline $1.8 \pm 0.3$ & $68.4 \pm 18.1$ & $119.5 \pm 30.8$ & $66.9 \pm 13.6$ & $38.5 \pm 10.9$ & $40.1 \pm 3.6$ \\
\hline
\end{tabular}

Values represent means \pm SEM. SEM, standard error of the mean.

on male fertility including, (I) the timing of disease onset relative to the semen analysis and (II) a lack of information concerning disease severity.

Most attempts to determine whether COVID-19 infection can influence semen quality have followed a cross sectional study design in which semen quality has been analysed in a group of post-COVID-19 patients, either in isolation (8) or in comparison with a cohort of control subjects that have not suffered infection with this virus (9). Even when an opportunity has arisen to conduct longitudinal studies of semen quality prior to and following COVID infection, the gaps between semen analysis and the onset of the disease (up to one year) and between recovery and the post infection analysis (at least 3 months) have been considerable (10). Furthermore, in all studies conducted to date, there has been little opportunity to define the precise relationship between the onset and severity of symptoms and any change in semen quality. In the attached case report, we address this important issue by exploring the unexpected infection of a semen donor within our scientific research program. By carefully monitoring semen quality up to the point where the donor was declared COVID-19 positive and then shortly after he had been declared COVID free, we reveal a surprisingly rapid and severe impact of viral infection on the semen profile, which then reversed within one spermatogenic cycle.

We present the following article in accordance with the CARE reporting checklist (available at https://tau. amegroups.com/article/view/10.21037/tau-21-935/rc).

\section{Case report}

The subject of this case report is a fit, healthy 55 -year-old triathlete with no other known co-morbidities, taking no medications or supplements, who consented to serve as a semen donor for the CellOxess research program on male infertility since 2018. This participant has provided written consent to be a part of this specific case report. As a result of his participation in CellOxess research, extensive background data on the subject's semen quality are available (Table 1) and are presented as annual quarterly averages in Figure 1. From 09/25/2018 to $12 / 05 / 2019$, his sperm counts and motilities were consistently within the normal range. Moreover, CASA analysis (Hamilton Thorne, Beverly, MA, USA) demonstrated that nearly $38.5 \% \pm 10.9 \%$ [mean \pm standard error of the mean (SEM)] of his spermatozoa were generally classified as rapid (moving with an average path velocity of more than $25 \mu \mathrm{m} / \mathrm{s}$ ) (13). Occasional measurements of oxidative DNA damage revealed a variable picture with a peak of $51 \%$ positivity in the October to December quarter of 2019. The CellOxess research program was on hold throughout 2020 during the height of the COVID-19 pandemic as staff and volunteers were under socially distanced "lockdown procedures". The program resumed in 2021 and in Figure 1, we present a longitudinal analysis of semen quality in this donor from 1/27/2021 onwards during which time, he contracted a COVID-19 infection.

The donors' first symptoms of COVID-19 infection were evident on 03/27/2021 and he had a positive COVID-19 test (Nasal Swab Rapid Antigen test by Solv and later RTPCR tests by Aegis Sciences Corporation, Nashville, TN, USA) on $03 / 31 / 2021$. The period of symptomatic disease was subsequently short, lasting only a few days such that by 04/09/2021 the patient was largely free of any overt signs of disease although he still returned a positive COVID result at that time. Just over a month later he returned two negative COVID-19 tests on 5/17/21 and 5/24/21. During the disease period, the subject did not require hospitalization but exhibited symptoms that would be considered moderate to serious, including fever $\left(101^{\circ} \mathrm{F}\right)$ in the first two days, fatigue and an inability to breath easily for 5 days after symptoms began. Unexpectedly, a semen sample taken on $04 / 21 / 2021$, approximately 3 weeks after the confirmed diagnosis of COVID-19 and around 2 weeks after the illness had resolved, revealed a state of azoospermia, which persisted for a month until 05/20/2021. Subsequent samples generated in early June $(06 / 03 / 2021$ and $06 / 10 / 2021)$ were severely oligozoospermic, with counts of $2.5 \times 10^{6} / \mathrm{mL}$ on both occasions. However, at this point, sperm motility $(69 \%$ and $60 \%$ respectively) had returned to normal (Figure 1). By the end of June (06/23/2021) both sperm count and motility were normal although levels of oxidative DNA damage (56.7\%) were still high, representing a $39.9 \%$ increase from his pre-COVID average (Figure 1). 
A Patient history pre-infection (2018-2020)

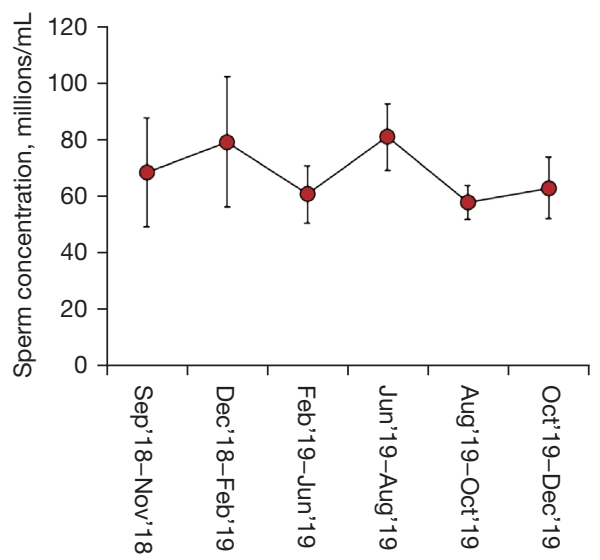

B

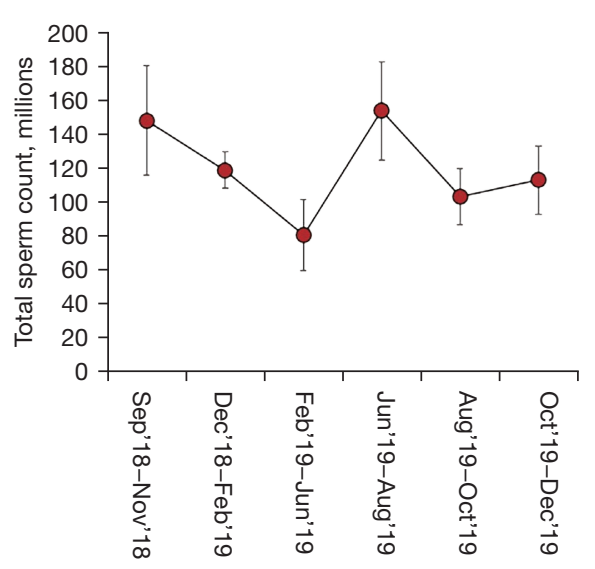

C

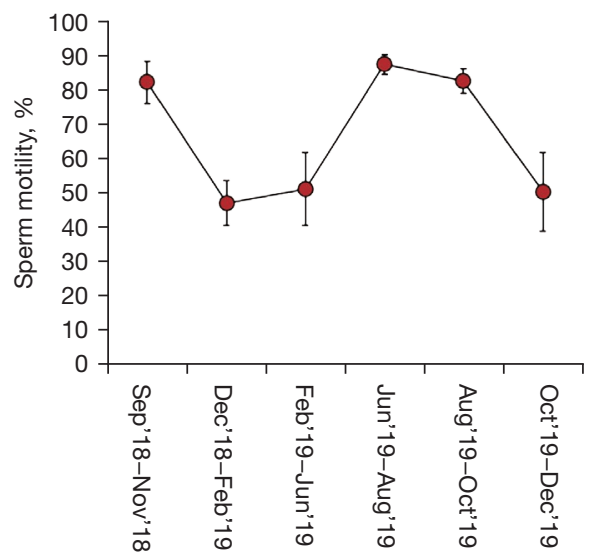

E

Patient data (2021)

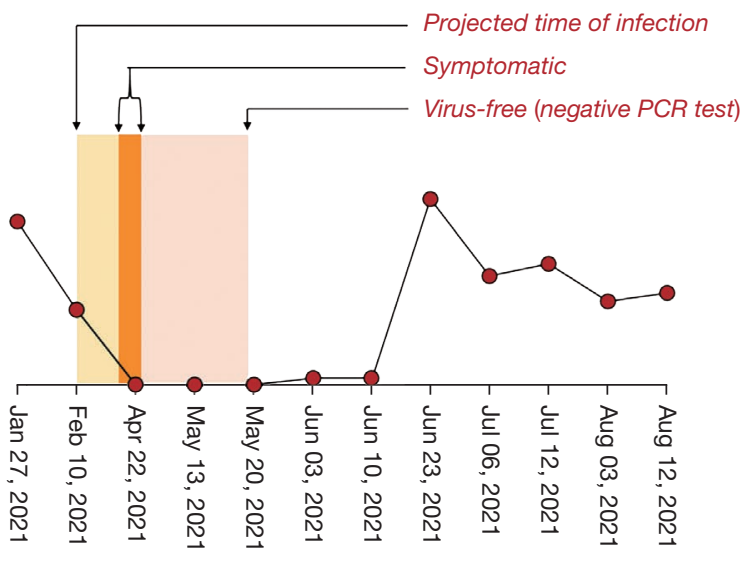

F

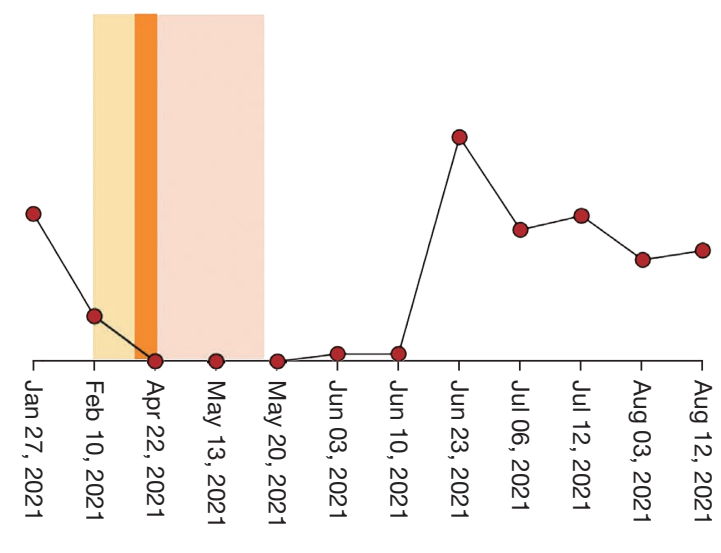

G

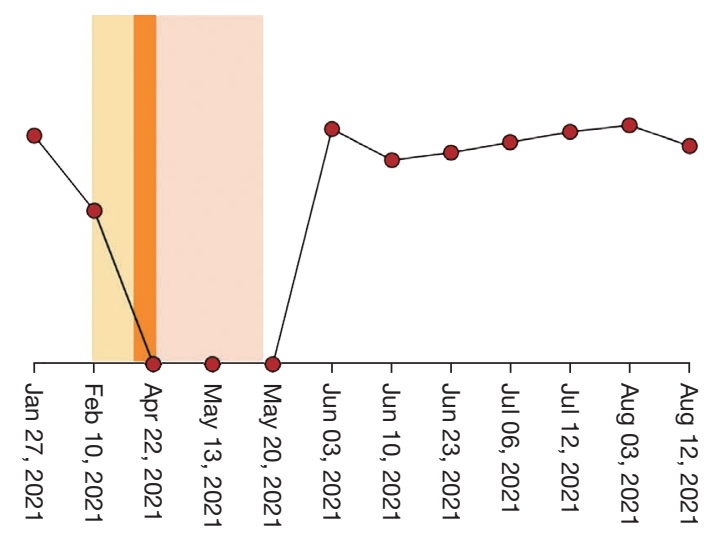



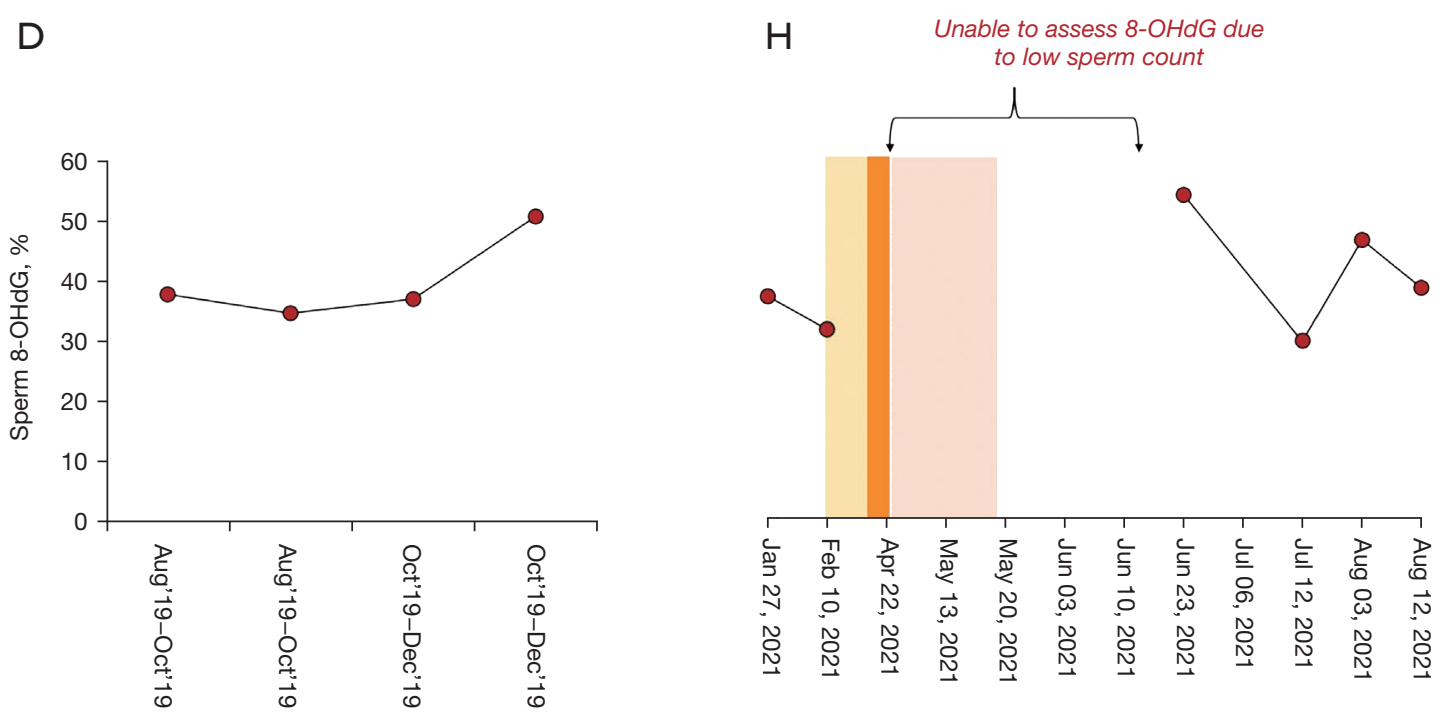

Figure 1 Longitudinal analysis of semen quality in a patient suffering from COVID-19 infection. (A-D) Dot plots to the left of the figure represent the mean $( \pm$ SEM) pre-infection semen parameters for samples collected between 09/25/18 and 12/05/19 for sperm concentration, total sperm count, and percentage motility ( $\mathrm{n}=5$ at each point in chronological order of submission, total sample submissions of $\mathrm{n}=30$ ). Oxidative DNA damage was measured via a modified 8-OHdG protocol $(11,12)$ between 10/04/19 and 11/21/19 (n=4). (E-H) Shaded areas indicate the stages and duration of infection. Dot plots to the right of the figure represent the pre- and post-infection semen values for sperm concentration, total sperm count, and percentage motility in chronological order of submission for samples collected between 01/27/21 and 08/12/21: total sample submissions of $(n=12)$. Oxidative DNA damage could not be measured from 04/22/21 through 06/23/21 due to assay limits on low sperm counts; total sample submissions analysed ( $\mathrm{n}=6$ ). SEM, standard error of the mean; 8-OHdG, 8-hydroxy-2'-deoxyguanosine.

Several aspects of this longitudinal study are noteworthy. First of all, the speed with which the patient was rendered azoospermic is impressive. Within 3 weeks of being diagnosed with the disease, spermatozoa were no longer detectable. Given that the combined length of spermatogenesis and epididymal maturation is around 11 weeks (78 days) in the human, we can retrospectively calculate that if the virus prevented spermatogonia from entering spermatogenesis then it must have attacked the germ line around early February when the patient was asymptomatic. Such a long viral incubation period in this patient is supported by the decline in semen quality observed between the end of January (01/27/2021) and the beginning of February (02/10/2021). During this time, sperm motility values declined from $67 \%$ to $45 \%$, while sperm counts reduced from 57.6 to $26.7 \times 10^{6} / \mathrm{mL}$. If this decline in semen is, indeed, indicative of early COVID-19 infection then it suggests that sperm production and maturation are extremely sensitive indicators of COVID-19 infection. However, this logic would also mean that the period of time between infection and the first appearance of symptoms would have been around 7 weeks. This is certainly longer than the generally accepted incubation period of 10-16 days and beyond the 99th percentile of 20 days (14). It is therefore possible that even though the virus can infect spermatogonia (15), COVID infection may have disrupted a later stage of spermatogenesis. Since the gap between the patient becoming symptomatic and the appearance of azoospermia is around 4 weeks, an alternative possibility is that the virus attacked spermatogenesis at the spermatocyte stage, which is known to be vulnerable to the kind of oxidative stress created by COVID infection (16). Ultimately, testicular biopsies from infected patients will be needed to confirm the precise relationship between the onset of infection and impaired spermatogenesis. In the meantime, we can conclude that the differentiation of male germ cells is exquisitely sensitive to COVID-19 infection.

In many ways, these data support the general concept that male fertility serves as a canary in the coal mine (17), providing important signals about the overall health status of a given individual in the absence of any other overt symptoms. The reduction in sperm motility observed during the early stages of infection could potentially be related to a direct effect of the virus on sperm viability via 
the stimulation of oxidative stress and apoptosis, subsequent to the inhibition of ACE2 and the dephosphorylation of AKT (1). A slight reduction in sperm number at this time may reflect the critical role of the epididymis in qualitycontrolling the ejaculate, by efficiently removing apoptotic cells from the epididymal lumen (18).

Using similar principles, we can predict that if spermatogenesis and sperm motility were re-established by late June $(06 / 23 / 2021)$, the process of sperm production could have been reinitiated at any time from early April, when the subject's symptoms had declined but he was still COVID-19 positive by PCR, to mid-May, when the patient became COVID negative, depending on the stage at which spermatogenic arrest was induced. This again emphasizes the exquisite sensitivity of spermatogenesis to COVID-19 infection. Furthermore, any disparity between viral presence and the reinitiation of spermatogenesis suggests that the suppression of sperm production is heavily influenced by the cytokine storm that accompanies COVID-19 infection, not just the presence of the virus itself. The fact that sperm motility was maximal during the earliest stages of recovery when the patient was still oligozoospermic, further suggests that epididymal function is not seriously compromised by COVID-19 infection: the testes are the target.

The speed with which subject went into, and recovered from an azoospermic state, may explain why some studies have reported severe impacts on male fertility accompanied by high levels of azoospermia (8) while others have found only minor disruptions to the conventional semen profile (9). It is clear from this case study, that overall semen quality will be heavily influenced by the timing of the sampling process relative to the onset of the disease.

\section{Conclusions}

To our knowledge this longitudinal analysis of a single subject immediately before, during and after COVID-19 infection is unique. It has provided a powerful insight into the sensitivity of the male reproductive system to COVID-19 infection revealing severe disruption of spermatogenesis in a patient who experienced a limited infectious episode. These results also emphasize the powerful ability of spermatogenesis to recover from viral infection and re-establish normal sperm production. The high levels of oxidative DNA damage observed in the spermatozoa as spermatogenesis was recovering may, however, be a warning that conception should not be attempted in the immediate aftermath of COVID-19 infection, lest it should impact the health and wellbeing of the offspring $(11,19)$. While limited to a case report, these unique data have revealed some fundamental features of COVID-19's impact on the male reproductive system that should be of value in clinically managing the impact of this pernicious disease on human fertility.

\section{Acknowledgments}

Funding: None.

\section{Footnote}

Reporting Checklist: The authors have completed the CARE reporting checklist. Available at https://tau.amegroups.com/ article/view/10.21037/tau-21-935/rc

Peer Review File: Available at https://tau.amegroups.com/ article/view/10.21037/tau-21-935/prf

Conflicts of Interest: All authors have completed the ICMJE uniform disclosure form (available at https://tau.amegroups. com/article/view/10.21037/tau-21-935/coif). The authors have no conflicts of interest to declare.

Ethical Statement: The authors are accountable for all aspects of the work in ensuring that questions related to the accuracy or integrity of any part of the work are appropriately investigated and resolved. All procedures performed in this study involving human participants were in accordance with the ethical standards of the institutional and/or national research committee(s) and with the Helsinki Declaration (as revised in 2013). Written informed consent was obtained from the patient for publication of this study and any accompanying images. A copy of the written consent is available for review by the editorial office of this journal.

Open Access Statement: This is an Open Access article distributed in accordance with the Creative Commons Attribution-NonCommercial-NoDerivs 4.0 International License (CC BY-NC-ND 4.0), which permits the noncommercial replication and distribution of the article with the strict proviso that no changes or edits are made and the original work is properly cited (including links to both the formal publication through the relevant DOI and the license). See: https://creativecommons.org/licenses/by-nc-nd/4.0/. 


\section{References}

1. Aitken RJ. COVID-19 and human spermatozoa-Potential risks for infertility and sexual transmission? Andrology 2021;9:48-52.

2. Zizza A, Recchia V, Aloisi A, et al. Clinical features of COVID-19 and SARS epidemics. A literature review. J Prev Med Hyg 2021;62:E13-24.

3. Aitken RJ. COVID-19 and male infertility: An update. Andrology 2022;10:8-10.

4. Machado B, Barcelos Barra G, Scherzer N, et al. Presence of SARS-CoV-2 RNA in Semen-Cohort Study in the United States COVID-19 Positive Patients. Infect Dis Rep 2021;13:96-101.

5. Li D, Jin M, Bao P, et al. Clinical Characteristics and Results of Semen Tests Among Men With Coronavirus Disease 2019. JAMA Netw Open 2020;3:e208292.

6. Koç E, Keseroğlu BB. Does COVID-19 Worsen the Semen Parameters? Early Results of a Tertiary Healthcare Center. Urol Int 2021;105:743-8.

7. Temiz MZ, Dincer MM, Hacibey I, et al. Investigation of SARS-CoV-2 in semen samples and the effects of COVID-19 on male sexual health by using semen analysis and serum male hormone profile: A cross-sectional, pilot study. Andrologia 2021;53:e13912.

8. Gacci M, Coppi M, Baldi E, et al. Semen impairment and occurrence of SARS-CoV-2 virus in semen after recovery from COVID-19. Hum Reprod 2021;36:1520-9.

9. Guo TH, Sang MY, Bai S, et al. Semen parameters in men recovered from COVID-19. Asian J Androl 2021;23:479-83.

Cite this article as: Gharagozloo P, Cartagena S, Moazamian A, Drevet JR, Somkuti S, Aitken RJ. Rapid impact of COVID-19 infection on semen quality: a case report. Transl Androl Urol 2022;11(1):110-115. doi: 10.21037/tau-21-935
10. Erbay G, Sanli A, Turel H, et al. Short-term effects of COVID-19 on semen parameters: A multicenter study of 69 cases. Andrology 2021;9:1060-5.

11. Vorilhon S, Brugnon F, Kocer A, et al. Accuracy of human sperm DNA oxidation quantification and threshold determination using an 8-OHdG immuno-detection assay. Hum Reprod 2018;33:553-62.

12. Drevet JR, Aitken RJ. Oxidative Damage to Sperm DNA: Attack and Defense. Adv Exp Med Biol 2019;1166:107-17.

13. Sloter E, Schmid TE, Marchetti F, et al. Quantitative effects of male age on sperm motion. Hum Reprod 2006;21:2868-75.

14. Dhouib W, Maatoug J, Ayouni I, et al. The incubation period during the pandemic of COVID-19: a systematic review and meta-analysis. Syst Rev 2021;10:101.

15. Duarte-Neto AN, Teixeira TA, Caldini EG, et al. Testicular pathology in fatal COVID-19: A descriptive autopsy study. Andrology 2022;10:13-23.

16. Pino JA, Osses N, Oyarzún D, et al. Differential effects of temperature on reactive oxygen/nitrogen species production in rat pachytene spermatocytes and round spermatids. Reproduction 2013;145:203-12.

17. Punjani N, Lamb DJ. Canary in the Coal Mine? Male Infertility as a Marker of Overall Health. Annu Rev Genet 2020;54:465-86.

18. Kwon J, Sekiguchi S, Wang YL, et al. The region-specific functions of two ubiquitin C-terminal hydrolase isozymes along the epididymis. Exp Anim 2006;55:35-43.

19. Aitken RJ, Bakos HW. Should we be measuring DNA damage in human spermatozoa? New light on an old question. Hum Reprod 2021;36:1175-85. 